\title{
Perception of Sleep in Recovering Alcohol-Dependent Patients With Insomnia: Relationship With Future Drinking
}

\author{
Deirdre A. Conroy, J.Todd Arnedt, Kirk J. Brower, Stephen Strobbe, Flavia Consens, Robert \\ Hoffmann, and Roseanne Armitage
}

\begin{abstract}
Background: Subjective and objective measures of poor sleep in alcoholic insomniacs predict relapse to drinking. Nonalcoholic insomniacs underestimate their total sleep time (TST) and overestimate their sleep onset latency (SOL) and wake time after sleep onset (WASO) compared with polysomnography (PSG). This study evaluated 3 hypotheses: (1) subjective SOL would predict frequency of future drinking; (2) participants would overestimate SOL and WASO and underestimate TST; and (3) higher amounts of over- and underestimates of sleep at baseline would predict worse drinking outcomes prospectively.

Methods: Participants $(N=18)$, mean age 44.6 years $( \pm 13.2)$, underwent an adaptation night and then 2 nights of PSG 3 weeks apart. They also provided morning estimates of SOL, WASO, TST, and sleep efficiency (SE). Following the baseline PSG, participants were followed over 12 weeks. A 2-way ANOVA (night $\times$ method of measuring sleep) compared results and regression analyses predicted drinking. Drinking outcomes were defined as number of days drinking (DD) and number of heavydrinking days (HDD) during 2 consecutive 6-week follow-up periods.

Results: Most participants $(72 \%)$ overestimated SOL by a mean of $21.3( \pm 36)$ minutes compared with PSG $[F(1,14)=7.1, p<0.03]$. Unexpectedly, $89 \%$ underestimated WASO by a mean difference of $48.7( \pm 49)$ minutes $[F(1,14)=15.6, p<0.01]$. Drinking during the first 6 -week study period was predicted by both subjective estimates of WASO and their accuracy, whereas drinking during the second 6-week period was predicted by both subjective estimations of sleep and rapid eye movement sleep latency.

Conclusion: Greater subjective accuracy of wakefulness at night provided by the patient predicted drinking during the study. Unlike nonalcoholic insomniacs, this alcoholic sample significantly underestimated WASO compared with PSG values. The predictive ability of sleep parameters depended on the selected measure of drinking outcomes and when outcomes were measured. Subjective sleep measures were better predictors of future drinking than corresponding PSG measures.
\end{abstract}

Key Words: Insomnia, Alcohol Dependence, Sleep Perception, Polysomnography, Sleep.

A LCOHOL HAS SOPORIFIC effects in nonalcoholic subjects, but after chronic use, the effects on sleep become deleterious. Within a night, alcohol has a biphasic effect on sleep. Initially, sleep onset latency (SOL), or the time it takes to fall asleep, is shortened and there is an increase in slow-wave sleep (SWS) in the first half of the night. In the second half of the night, sleep quality deteriorates and there are more awakenings (Rundell et al., 1977). As alcohol use becomes more chronic, tolerance to the sedating effects develops and individuals require greater amounts of alcohol more frequently to derive the same sleep-promoting effects. As the neurochemical systems of the brain adapt, sleep-promoting systems adapt their

From the University of Michigan, Ann Arbor, Michigan.

Received for publication May 10, 2006; accepted August 7, 2006.

This research was supported by Grants T32AA07477, K24AA00304, and MO1 RR00042.

Reprint requests: Deirdre A. Conroy, PhD, University of Michigan, 2025 Traverwood Drive, Ann Arbor, MI 48105; Fax: 734-998-7992; E-mail:daconroy@umich.edu

Copyright (C) 2006 by the Research Society on Alcoholism.

DOI: 10.1111/j.1530-0277.2006.00245.x responses to the chronic alcohol administration and sleep propensity is dampened (Brower, 2003).

Sleep continues to be severely disturbed in early recovery from alcohol dependence. A review of studies estimated that $36 \%$ to $72 \%$ of participants in early recovery from alcohol dependence complained of insomnia (Brower, 2001). Cohn et al. (2003) found that 91\% of alcoholic participants had sleep disturbance as measured by a well-validated measure of sleep disturbance: the Pittsburgh Sleep Quality Index (Buysse et al., 1989; Cohn et al., 2003). These findings have been supported by earlier experimental studies documenting complaints of sleep disturbance during early recovery (Baekeland et al., 1974; Brower et al., 2001; Caetano et al., 1998; Foster et al., 2000; Mello and Mendelson, 1970). Both subjective complaints of poor sleep and disrupted sleep measured by polysomnography (PSG) have been shown in naturalistic treatment outcome studies to predict relapse to drinking among alcohol-dependent participants. For example, Brower et al. (1998) found that SOL, whether measured by PSG or self-report, predicted return to any drinking within 5 months of starting treatment. 
Understanding how a patient perceives his or her sleep is important because it may have an impact on the patient's future behaviors. It is well established that nonalcoholic participants with insomnia are subjectively inaccurate: they underestimate their total sleep time (TST) and overestimate SOL and the time they are awake in the night after they fall asleep, i.e., wake time after sleep onset (WASO), compared with same-night objective results of PSG (Baekeland and Hoy, 1971; Carskadon et al., 1976; Frankel et al., 1976; Kales and Kales, 1984; Monroe, 1967) This misperception may contribute to sleep-disruptive behaviors and dysfunctional beliefs about one's sleep that ultimately perpetuate factors in the course of the insomnia. Misperception of sleep in a recovering alcohol-dependent individual with insomnia may be additionally detrimental. If a recovering alcoholic patient perceives his or her sleep to be poor, then this perception may contribute to a relapse to drinking.

Only one study has looked at the relationship between subjective and objective assessments of sleep in a population of alcoholic insomniacs (Currie et al., 2004). The authors directly compared subjective reports of insomnia (including sleep logs, questionnaires, and reports from the patient's partner and clinician) to wrist actigraphy, which served as the objective measure of insomnia. Results from 1 week of simultaneous sleep log and actigraphy data revealed that participants overestimated SOL by a mean of 16 minutes, but accurately perceived sleep efficiency (SE) and TST. In contrast to nonalcoholic insomniacs, however, alcoholic participants underestimated WASO by a mean of 1 hour. In other words, after falling asleep, they perceived that they slept better than they actually did. This finding was unexplained.

To further characterize an individual's estimate of sleep in this study, we used a sleep parameter that measures the relationship between subjective estimates of sleep parameters (obtained via sleep logs) and corresponding objective sleep measures (obtained via PSG) for the same night of sleep. This measure was termed the objective sleep time estimated (OSE) score by Edinger and Fins (1995). The score, expressed as a percentage, provides a quantitative measure of accuracy that can be used as a standard method to compare the accuracy of perceptions among different sleep variables and subject groups. In particular, it has been used to distinguish primary insomnia from secondary insomnia and to compare insomnia subgroups (Means et al., 2003). It has also been used to compare subjective estimates of sleep to actigraphy data in recovering alcoholic patients postwithdrawal (Currie et al., 2004).

The present study extends the existing literature in several ways. First, no study has conducted a quantitative comparison of subjective sleep measures with PSG, the gold standard of objective sleep measurement, in alcoholdependent insomnia participants. Actigraphy is only able to detect the presence or absence of movement and therefore is an indirect measure of sleep. By contrast, PSG provides direct quantitative measures of sleep architecture (sleep stages) and continuity (e.g., SOL, TST, WASO). In general, the relationship between objective and subjective sleep measures is poorly understood, but studies are particularly sparse among individuals with a history of alcohol dependence because few insomnia trials include alcohol-dependent participants.

This study includes only alcoholic participants with a clinical diagnosis of insomnia, whereas previous sleep studies of alcoholic-dependent participants selected participants meeting criteria for alcohol dependence only (cf. Brower et al., 1998). No previous study has examined either the subjective accuracy of sleep or the relationship between the accuracy of these perceptions and future drinking behavior in patients with alcohol dependence and comorbid insomnia. Additionally, this study adds to the existing literature on impaired sleep and future drinking because, instead of utilizing only a single dichotomous measure of relapse (i.e., return to any drinking) over one time interval, we utilized 2 drinking frequency measures [number of drinking days (DD) and number of heavy drinking days(HDD)] and assessed when drinking occurred (during 2 consecutive 6-week follow-up periods). We hypothesized that subjective SOL in alcohol-dependent participants with insomnia would predict drinking during both follow-up periods. Second, we hypothesized that participants would overestimate SOL and WASO, and underestimate TST, and that the greater the subjective/objective discrepancies at baseline (i.e., less accurate OSE scores), the worse drinking outcomes would be during the follow-up periods.

\section{MATERIALS AND METHODS}

\section{Participants}

Twenty-one (10 women) insomnia participants in early recovery from alcohol dependence were recruited from a local outpatient alcohol treatment facility, or the community, by way of advertising. All of these individuals were taking part as paid volunteers in a randomized-controlled trial of the effects of gabapentin versus placebo on relapse to alcohol use.

\section{Procedures}

Participants responded to flyers advertising a study for individuals who "have problems sleeping" and who "use alcohol to help them sleep." Interested participants telephoned the study's research coordinator and underwent an initial screening interview. The interview consisted of questions relating to quantity and frequency of drinking, date of last alcohol use, sleeping habits, medications, and psychiatric history. Participants needed to express a desire to stop drinking or a willingness to abstain from use of alcohol and other drugs of abuse (except nicotine) throughout their participation in the study. A study investigator (S.S.) evaluated data collected from a telephone screening interview and determined whether or not an interested participant was appropriate for further screening. At that time, a date and time were set for a more comprehensive screening appointment. At the time of appointment, participants verbally agreed to have a breath alcohol content measurement taken. Written informed consent for additional screening and research participation was obtained only if blood alcohol levels were less than $0.05 \%$. 
Participants were excluded if they met the following criteria: were $<18$ years of age; met DSM-IV criteria for panic disorder, social phobia, generalized anxiety disorder, posttraumatic stress disorder, major depression, bipolar disorder, anorexia nervosa, or bulimia nervosa in the past month; met criteria for dependence on any psychoactive substance other than alcohol (except nicotine) in the past 1 month; had a medical condition or chronic pain syndrome that caused insomnia; had insomnia that was associated with sleep apnea (Respiratory Disturbance Index $>10$ ) or periodic limb movements (periodic limb movement index with arousals >15) (determined via PSG); and required treatment with medications known to affect sleep such as mirtazepine, trazodone, tricyclic antidepressants, neuroleptics, sedative hypnotics, stimulants, centrally acting antihistamines or antihypertensives, oral corticosteroids, or theophylline. Patients were also excluded if they were taking mood stabilizers or other antidepressants only if there had been a recent change in the past 2 months in the medications or a change was anticipated during the course of the study or if the study investigator determined the medication or its underlying disorder was contributing to their insomnia.

Of the 35 participants who qualified for insomnia and who underwent an in-person screening process, 14 were excluded: 7 who were lost to follow-up before completing the screening process, 1 who was referred for residential treatment, 1 who could not maintain abstinence from alcohol use through the screening period, 1 who did not meet alcohol dependence criteria, 2 who had positive urine drug screens for cocaine, 1 who was excluded based on PSG results indicating severe sleep apnea, and 1 who was excluded based on PSG results indicating periodic leg movement sleep disorder. In summary, 2 of 14 excluded subjects, or $\sim 14 \%$, were excluded due to other sleep disorders besides primary insomnia.

Participants reported persistent insomnia for at least 1 week postabstinence with a score on the revised version of the Clinical Institute Withdrawal Assessment for Alcohol (CIWA-A) of $<8$ (Sullivan et al., 1989) to ensure that persisting insomnia was not due to acute alcohol withdrawal. They were excluded if they rated their insomnia as "much improved" or "very much improved" on the Clinical Global Impression Scale after 1 week of abstinence (Guy, 1976). Insomnia was assessed and diagnosed by the authors (K.B. or S.S.) using the Sleep Disorders Questionnaire (Douglass et al., 1994), the Sleep Problems Questionnaire (Jenkins et al., 1988), and the Insomnia Interview Schedule (Morin, 1993). Participants meeting the above criteria were scheduled to return for a baseline sleep assessment.

After completing the study instruments and measures in the screening visit, participants were followed for a 7- to 14-day baseline period. The purpose of this period was to promote and assess for abstinence and to document persisting insomnia before randomization. Participants were provided with a sleep log and asked to record their sleep schedule. Participants also received a nightly single-blind placebo capsule during baseline as a lead-in to the 6-week medication trial.

At the conclusion of 1 week of abstinence, each eligible participant was admitted to the University of Michigan General Clinical Research Center (GCRC) sleep laboratory and underwent nocturnal PSG recordings on 2 consecutive nights. Upon arrival for each study night, they were given a test for breath alcohol content and were either excluded or rescheduled for any positive result. The first night was an adaptation night to rule out occult sleep disorders. After excluding subjects for an occult sleep disorder, the data from this night were not used in any of the analyses. Therefore, the next night of PSG was referred to as study "Night 1." Participants generally arrived in the laboratory at approximately 9:00 PM and left between 7:00 and 8:00 AM the next day. Participants completed sleep logs before leaving the laboratory each morning.

Following Night 1, qualified participants were randomized to either placebo $(n=11)$ or gabapentin $(n=10)$ for 6 weeks as part of a double-blind randomized-controlled trial. After 3 more weeks in the study, participants returned for their final night of PSG, referred to as "Night 2." Upon morning awakening after each night, participants provided estimates of their SOL, WASO, and TST in their sleep log.

Of the 21 subjects who qualified, data for 2 participants were not included because they did not report their subjective assessment of sleep after Night 1. Data for another subject could not be made available, leaving a total of 18 subjects for analysis.

\section{Primary Outcome Measures}

Objective Sleep. Objective sleep measurement consisted of an electroencephalogram (C3/A2 EEG), referential electrooculogram (EOG) with electrodes placed at the outer canthus of each eye, one immediately above the other just below the horizontal plane, to record both horizontal and vertical slow and rapid eye movements (REM), submental electoromyogram (EMG), respiratory monitoring (nasal-oral airflow monitor, abdominal and chest monitors of respiratory effort, and ear or finger oximetry), electrocardiogram, and EMG of the anterior tibialis muscle (to document periodic leg movements). Data were recorded using a paperless system (Telefactor Corp., Grass Technologies, West Warwick, RI). The primary PSGdependent variables were TST, SOL, WASO, and SE. In addition, the percentage of time spent in Stages 1, 2, 3, and 4 and REM sleep were determined, as was REM onset latency. Polysomnography studies were scored by standard criteria (Rechtschaffen and Kales, 1968).

Subjective Sleep Estimates. Subjective sleep was measured via sleep logs that were completed upon morning awakening from Night 1 and Night 2 in the laboratory. Estimates used in for this study included SOL, WASO, and TST. Participants did not provide estimates of SE. Rather, subjective SE for this study was calculated by a research assistant, based on the ratio of subjectively estimated TST divided by reported time in bed, and multiplied by 100 . Thus, $\mathrm{SE}$ is expressed as a percentage, and lower numbers are indicative of greater sleep discontinuity.

Accuracy Measurements. To calculate the subjective accuracy of each sleep parameter, a variation of the OSE (Edinger and Fins, 1995) was used. This measure is derived by using the following formula: $\mathrm{OSE}=($ subjective parameter/objective parameter $) \times 100 \%$. In this formula, the subjective parameter is the sleep log estimate and the objective measure is the PSG for the parameter being examined. The OSE score for SE reflects the ratio of subjective SE that was calculated by a research assistant divided by the SE value determined polysomnographically. An OSE score of $100 \%$ indicates perfect agreement between objective and subjective estimates; OSE $<100 \%$ indicates subjective underestimation relative to PSG; and $>100 \%$ indicates subjective overestimation.

Alcohol Consumption. Daily alcohol consumption was obtained using the Timeline Follow-Back Interview (TLFB) (Sobell et al., 1988). Frequency of any drinking was defined by the number of DD during a 6-week period. Frequency of heavy drinking (which combines frequency and quantity) was defined as the number of HDD during a 6 -week period. Heavy drinking was defined as $>4$ standard drinks in a day for women and $>5$ standard drinks in a day for men. A standard drink is equivalent to $12 \mathrm{oz}$ of beer, $5 \mathrm{oz}$ of table wine, or 1 to $1.5 \mathrm{oz}$ of liquor (NIAAA, 2005).

\section{Data Analyses}

A 2-way ANOVA of the sleep assessment method (sleep log vs PSG) versus night (Night 1 vs Night 2) was computed for each of the dependent variables SOL, WASO, TST, and SE, to examine withinnight and between-night differences in sleep parameters. Paired $t$ tests were also computed. Regression analyses were computed to examine the relationships between Night 1 objective and subjective measures of baseline sleep and subsequent alcohol consumption during the 2 consecutive follow-up periods. The first 6-week period 
included medication treatment, and the second 6-week period did not. Statistical analyses were performed by using SPSS (Version 13.0).

\section{RESULTS}

\section{Sample Demographics}

Eighteen participants ( 9 females) completed Night 1 only. Fifteen participants ( 7 females) completed both Night 1 and Night 2. Before entering the study, participants reported having a mean $(\mathrm{SD})$ of $31.2( \pm 13) \mathrm{DD}$ and $21.2( \pm 16) \mathrm{HDD}$ in the past 90 days. Participants were abstinent in the week before the randomization trial, except for 1 subject who had 2 drinks 4 days before the trial began. Independent sample $t$ tests revealed no differences between the gabapentin group and placebo group on any of the subjective or objective sleep measures for the pretreatment night or posttreatment night; therefore, these groups were collapsed.

\section{Objective Sleep by PSG from Night 1 to Night 2}

The mean sleep parameters for Night 1 versus Night 2 are displayed in Table 1. A paired sample $t$ test was conducted to evaluate the difference between study nights. There were no differences between Night 1 and Night 2 sleep variables.

\section{Subjective Versus Objective Accuracy}

Using paired sample $t$ tests and correlation coefficients, the results comparing subjective estimates $(\log )$ and objective (PSG) sleep parameters within each night are shown in Table 2. A 2-way within-groups analysis of variance was conducted to explore the impact of night (Night 1 vs Night 2) and assessment (objective PSG data vs subjective sleep $\log$ ) on subjective estimations only.

On Night 1 , there was a statistically significant main effect for SOL assessment $[F(1,14)=7.1, p<0.03]$. The partial $\eta^{2}$ statistic (0.34) indicated a large effect size (Cohen, 1988). Post hoc comparison using a paired sample $t$ test indicated that the mean score for Night 1 subjective assessment of SOL (48.1 \pm 49.2$)$ minutes was significantly higher than the objective data, $26.8( \pm 29.9)$ minutes. Most $(72 \%)$ participants overestimated SOL compared with PSG $(p<0.05)$, with a group mean of $21.3( \pm 36)$ minutes and a mean of 30.5 $( \pm 36.7)$ minutes among the overestimators.

Table 1. Objective Sleep Measures

\begin{tabular}{lccc}
\hline Sleep measure & Night $1(n=15)$ & Night 2 & $p$ Value \\
\hline Total sleep time (min) & $317.5( \pm 47.4)$ & $303.5( \pm 68.8)$ & 0.27 \\
Sleep onset latency (min) & $28.4( \pm 32.0)$ & $35.3( \pm 45.9)$ & 0.39 \\
Sleep efficiency (\%) & $77.4( \pm 7.1)$ & $75.7( \pm 13.9)$ & 0.63 \\
Stage 1 (\%) & $14.1( \pm 10.4)$ & $13.0( \pm 9.6)$ & 0.47 \\
Stage 2 (\%) & $55.7( \pm 10.6)$ & $59.2( \pm 8.8)$ & 0.27 \\
Stage 3/4 (\%) & $11.2( \pm 7.6)$ & $12.8( \pm 9.3)$ & 0.45 \\
REM (\%) & $19.0( \pm 6.5)$ & $14.9( \pm 5.8)$ & 0.11 \\
REM latency (min) & $97.8( \pm 46)$ & $110.9( \pm 56.7)$ & 0.43 \\
Wake after sleep onset (min) & $92.4( \pm 32.3)$ & $96.9( \pm 57.5)$ & 0.77 \\
\hline
\end{tabular}

REM, rapid eye movement.
Table 2. Comparison of Subjective and Objective Measures of Sleep

\begin{tabular}{lccrc}
\hline & $\begin{array}{c}\text { Sleep log } \\
(\text { SD })\end{array}$ & $\begin{array}{c}\text { PSG } \\
( \pm \text { SD })\end{array}$ & $\begin{array}{c}\text { Mean } \\
\text { discrepancy } \\
( \pm \text { SD })\end{array}$ & $\begin{array}{c}\text { Paired } \\
\text { samples } \\
t \text { tests }\end{array}$ \\
Variables & & & & \\
\hline Night 1 $(n=18)$ & & & & \\
SOL (min) & $48.1( \pm 49)$ & $26.8( \pm 30)$ & $-21.3( \pm 36)$ & $-2.5^{*}$ \\
WASO (min) & $38.2( \pm 41)$ & $86.9( \pm 35)$ & $48.7( \pm 49)$ & $4.2^{* *}$ \\
SE (\%) & $76.09( \pm 15)$ & $79.0( \pm 8)$ & $2.9( \pm 14)$ & 0.9 \\
TST (min) & $324.0( \pm 92)$ & $328.94( \pm 51)$ & $4.97( \pm 75)$ & 0.3 \\
Night 2 ( $n=15)$ & & & & \\
SOL (min) & $62.5( \pm 58)$ & $35.3( \pm 46)$ & $-17.1( \pm 36)$ & -2.0 \\
WASO (min) & $48.3( \pm 58)$ & $96.91( \pm 58)$ & $42.5( \pm 78)$ & $3.1^{*}$ \\
SE (\%) & $70.7( \pm 24)$ & $75.7( \pm 14)$ & $2.8( \pm 20)$ & 0.5 \\
TST (min) & $286.9( \pm 103)$ & $303.5( \pm 68)$ & $5.1( \pm 73)$ & 0.3 \\
\hline
\end{tabular}

${ }^{*} p<0.05 ;{ }^{* *} p<0.001$.

SOL, sleep onset latency; WASO, wake time after sleep onset; SE, sleep efficiency; TST, total sleep time; PSG, polysomnography.

There was a statistically significant main effect for WASO assessment $[F(1,14)=15.6, p<0.01]$. The effect size was large (partial $\eta^{2}=0.53$ ). The mean subjective assessment of WASO was $38.2( \pm 40.7)$ minutes, which was unexpectedly lower than the objective data, $86.9( \pm 34.5)$ minutes. The majority $(89 \%)$ of the participants underestimated WASO by a mean difference of $48.7( \pm 49.14)$ minutes for the overall group and $58.8( \pm 41.5)$ minutes among the underestimators. Assessments of TST or SE by log estimates did not differ significantly from PSG values.

On Night 2, 11 of 15 participants $(73 \%)$ overestimated SOL by a mean of $17.09( \pm 35.5)$ minutes compared with PSG, although the effect was not significant $(p<.07)$. Fourteen of 15 participants $(93 \%)$ underestimated WASO by $42.54( \pm 78.3)$ minutes $(p<.02)$ (Table 2$)$. Assessments of TST or SE by log estimates did not differ significantly from PSG values.

Median OSE scores were used to describe OSE scores, because Kolmogorov-Smirnov tests of normality revealed that 6 of the 8 OSE scores (except for Night 2 TST and SE) were not normally distributed. These scores across the 2 study nights are displayed in Fig. 1. Participants consistently underestimated WASO, with a median OSE score of $29 \%$ on Night 1 and $38 \%$ on Night 2 . The median OSE scores for SOL were 156 and $138 \%$, indicating overestimation on both nights. Participants were generally accurate in their estimation of the number of hours they slept (98 and 106\%) and how well they slept, as indicated by SE ( 97 and 104\%). The degree of over- or underestimation within each variable did not differ from Night 1 to Night 2. A split-plot analysis of variance revealed that there was no medication effect on OSE scores for SOL, TST, SE, or WASO on Night 1 or Night 2.

\section{Relationship with Drinking}

A series of multiple regressions were conducted to examine the relationship between Night 1 objective sleep parameters and subjective estimates of sleep and drinking 


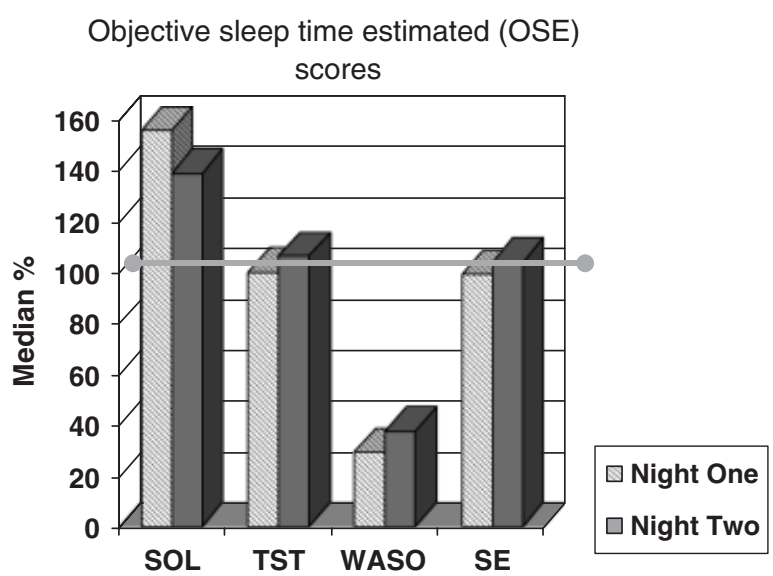

Fig. 1. Objective sleep time estimated scores across following 2 nights of study for each sleep variable. Greater than $100 \%$ represent overestimation of the respective variable and less than $100 \%$ represent underestimation of the respective variable. The horizontal bar at $100 \%$ represents perfect accuracy. Objective sleep time estimated scores demonstrate a consistent underestimation of WASO both across subjects and across the 2 study nights. SOL, sleep onset latency; WASO, wake time after sleep onset; SE, sleep efficiency; and TST, total sleep time; OSE, objective sleep time estimated.

variables after controlling for previous drinking (number of DD or HDD) and treatment group (i.e., either gabapentin or placebo). Baseline drinking frequencies and medication group were entered as control variables into the regression analyses to predict subsequent alcohol consumption from baseline sleep parameters. Initial analyses using logistic regression to predict any drinking versus no drinking revealed no significant relationships between sleep variables and drinking and therefore HDD and DD were analyzed as outcome measures.

The more accurate participants were at detecting periods of wakefulness in their sleep during the study, the more alcohol they consumed and the more frequently they drank. Frequency of heavy drinking (HDD) during the first 6-week follow-up period was predicted by both sleep-log subjective estimates and OSE scores of WASO $\left(R^{2}=0.69\right.$, $p<0.02$, and $R^{2}=0.49, p<0.02$, respectively). Similarly, frequency, expressed as DD during this phase, was predicted by sleep-log subjective estimates of WASO and accuracy $\left(R^{2}=0.53, p<0.03\right.$, and $R^{2}=0.55, p<0.02$, respectively). Overestimation of SOL was not predictive of drinking during the outpatient study phase.

The single objective variable that significantly predicted relapse was REM sleep latency $\left(R^{2}=0.59, p<0.05\right)$. After controlling for treatment group and previous days drinking, baseline REM latency predicted HDD during the first 6 weeks, such that the longer the REM latency, the more the HDD. Polysomnography measures of SOL, TST, SE, WASO, REM sleep percentage, and sleep stages 1 to 4 did not predict any drinking outcomes.

In the second 6 weeks, the following variables were predictive of HDD: OSE scores of SOL $\left(R^{2}=0.67, p<0.03\right)$, subjective estimates of SOL $\left(R^{2}=0.58, p<=0.05\right)$, OSE scores of WASO $\left(R^{2}=0.53, p<0.05\right)$, and OSE scores of
SE $\left(R^{2}=0.70, p<0.02\right)$. Days drinking were predicted by OSE scores of SE $\left(R^{2}=0.51, p<0.05\right)$, subjective estimations of TST $\left(R^{2}=0.79, p<0.02\right)$, and the accuracy (OSE scores) of TST estimations $\left(R^{2}=0.49, p=0.05\right)$.

\section{DISCUSSION}

The primary aim of the study was to evaluate the association between objectively measured sleep and the subjective estimates of sleep in a sample of alcohol-dependent participants with insomnia. The secondary aim was to examine objective and subjective indices of sleep as predictors of drinking outcomes during a 12-week follow-up period, divided into 2 consecutive 6-week blocks. There were 4 main study findings. First, as expected and similar to nonalcoholic insomnia participants, most participants overestimated SOL. However, unlike nonalcoholic insomnia participants, this alcoholic sample significantly underestimated WASO compared with actual PSG values. Second, subjective sleep measures (SOL and WASO) were better predictors of future drinking than corresponding PSG measures. Third, the predictive ability of baseline sleep parameters depended on the selected measure of drinking outcomes and when the outcome was measured (i.e., during the first or second 6-week follow-up period). Finally, there were no changes in objective or subjective sleep measures, including OSE scores, from Night 1 to Night 2.

\section{Subjective Estimates}

Subjective sleep estimates, collected from sleep logs, and their accuracy, reported as OSE percentages, revealed a consistent underestimation of WASO among participants and across the study nights (Fig. 1). These findings, although counter to our original hypothesis, are consistent with Currie et al. (2004), who found that alcohol-dependent insomnia participants similarly underestimated WASO. In that study, the median WASO OSE scores were $70.7 \%$, which was higher than our findings of 29 and $38 \%$ on Night 1 and Night 2, respectively. Two factors may account for these discrepancies. First, Currie et al. (2004) used wrist actigraphy, which measures activity level, an indirect measure of sleep, rather than PSG and, therefore, may have detected less wakefulness during the night. Actigraphs do underestimate awakenings if no motor activity accompanies the arousal (Hoffmann et al., 2004). Second, participants in the Currie study were abstinent for an average of $15.7( \pm 20.2)$ months, whereas the mean duration of abstinence in the present study was only 20 days. Thus, changes in objective sleep over prolonged abstinence may be associated with changes in subjective sleep, but little is known about this relationship. It is also possible that participants become more aware of wakefulness in the night with prolonged abstinence, a well-documented characteristic of chronic insomnia participants (Rosa and Bonnet, 2000). Overall, these results suggest that alcohol-dependent participants in early recovery may not initially report 
wakefulness in the night, even though sleep disruption is evident on the PSG. Additionally, they may be more likely to report difficulty falling asleep than waking during the night. This may be due, at least in part, to preexisting trait characteristics such as heightened anxiety sensitivity.

The mechanisms underlying the evolution of the insomnia complaint in alcohol-dependent participants are not known. As alcohol affects many of the same areas of the brain that are involved in the initiation and regulation of sleep (for a review, see Brower, 2001, 2003), it is tempting to speculate that neurotoxicity from chronic alcohol use may be affecting the memory for, and perceptual distinction of, wakefulness and sleep upon morning awakening. The particular pattern of WASO underestimation observed in this study may be a characteristic of insomnia in early recovery among this subpopulation.

\section{Predicting Relapse}

Overestimation of SOL on the sleep logs and accuracy by OSE percentages of SOL were predicted drinking only during weeks 7 to 12 of the study, Although it has been well documented that subjectively reported SOL predicts relapse in alcohol-dependent participants (Brower et al., 1998, 2001; Drummond et al., 1998; Foster et al., 1998; Foster and Peters, 1999; Skoloda et al., 1979), the current study focused on additional variables and their relationship between drinking behaviors during different follow-up periods. Our findings suggest that difficulty falling asleep may present the greatest risk for relapse once the patient finishes an episode of treatment for alcohol dependence.

Subjective estimation of WASO on sleep logs as well as OSE WASO percentages were better predictors of drinking during the first 6 weeks. Wake time after sleep onset has not been shown to predict relapse in previous studies of alcohol-dependent participants (Brower et al., 1998; Foster and Peters, 1999). However, the results from the present study may differ from previous studies because participants enrolled in this study were selected because of an insomnia complaint, not simply because they were recovering alcohol-dependent patients. Furthermore, the sample population differs from those studied in other naturalistic studies (Cohn et al., 2003; Foster et al., 1998; Foster and Peters, 1999) in that it reflects only those alcohol-dependent patients with insomnia in the absence of other occult sleep disorders (e.g. sleep apnea), use other drugs of abuse, and comorbid disorders. Therefore, one must consider the external validity and how this sample differs from a "typical" alcoholic population. Nevertheless, these results suggest that different rates and degrees of relapse may be associated with the particular type of insomnia complaint in recovering alcoholic patients.

Overall, this study suggests that subjective impressions of sleep were better predictors of relapse than PSG measures. Unlike previous studies (Brower et al., 1998; Drummond et al., 1998), objectively determined SOL by PSG did not predict relapse. The percent of SWS also did not predict relapse, contrary to some previous findings (Aldrich et al., 1999; Allen et al., 1977), but consistent with others (Brower et al., 1998; Gann et al., 2004; Gillin et al., 1994).

The only objective measure that predicted relapse was REM sleep latency. However, these findings indicate an association between a long REM sleep latency on Night 1 and the quantity of DD during the second 6-week followup period. This finding is inconsistent with previous studies (Aldrich et al., 1994; Allen and Wagman, 1975; Brower et al., 1998; Clark et al., 1998; Freemon, 1982; Gann et al., 2001; Gillin et al., 1994), which showed that a short REM latency predicted relapse.

There were limitations to this study. First, there were no direct comparisons with age-matched and sex-matched nonalcoholic insomniac controls. Second, the sample size was small. Finally, because it has been suggested that cognitive profile (Bastien et al., 2003), personality (Dorsey and Bootzin, 1997), and sleep-related beliefs (Edinger et al., 2000) impact subjective assessments of sleep, additional sleep-related questionnaires may have provided more information about a given participant's sleep-related thoughts and behaviors. A strength of the study was that despite the small sample size, the subjective findings were relatively strong. Attention to subjective reports in a clinical setting and questionnaire data in the research setting should be emphasized.

\section{Clinical Implications}

The results of the present study have important implications for understanding and managing alcoholic participants with insomnia. First, such patients may be prone to overestimate initial insomnia and underestimate middle insomnia. When targeting insomnia with treatment, therefore, both initial and middle insomnia should be considered even though one type of insomnia may generate the complaint. Second, both subjective estimates of sleep and their accuracy compared with objective sleep measures may predict future drinking better than objective measures alone. Therefore, the use of sleep logs and subjective sleep assessments may be a useful tool in the clinic. Moreover, normalization of PSG in the presence of persisting sleep complaints may be an insufficient goal of treatment. Nevertheless, health care professionals working with recovering alcohol-dependent participants with insomnia should consider referring their participants for a more extensive sleep evaluation if initial treatment efforts despite continuing abstinence fail to rule out sleep apnea and periodic limb movement disorder.

In conclusion, we found that alcohol-dependent insomnia participants early in recovery overestimate SOL and underestimate the amount of wakefulness in sleep. In addition, the study highlights a potential distinction between insomnia complaints in recovering alcoholic patents versus nonalcoholic participants. This pattern of 
sleep perception may be a signature characteristic of this insomnia subpopulation and directly contribute to relapse, but this requires further study.

\section{ACKNOWLEDGMENTS}

We thank Anne Buu, PhD, PhD, Research Investigator, Substance Abuse Section, Department of Psychiatry, University of Michigan, for her statistical consultation, and Karen Kairys, MPH, RN, CARN, Clinical Care Coordinator, UM Addiction Treatment Services, for her assistance with data collection and retrieval.

\section{REFERENCES}

Aldrich MS, Brower KJ, Hall JM (1999) Sleep-disordered breathing in alcoholics. Alcohol Clin Exp Res 23:134-140.

Aldrich MS, O’Neal E, Eiser A, Kroll P, Brower K, Shipley J (1994) Slow wave sleep decrement and relapse tendency in alcoholics in treatment. Sleep Res 23:185.

Allen R, Wagman A (1975) Do sleep patterns relate to the desire for alcohol. Adv Exp Med Biol 59:495-508.

Allen R, Wagman A, Funderburk F (1977) Slow wave sleep changes: alcohol tolerance and treatment implications. Adv Exp Med Biol 85A:629-640.

Baekeland F, Hoy P (1971) Reported vs recorded sleep characteristics. Arch Gen Psychiatry 24:548.

Baekeland F, Lundwall L, Shanahan TJ, Kissin B (1974) Clinical correlates of reported sleep disturbance in alcoholics. Q J Stud Alcohol 35:1230-1241.

Bastien C, Fortier-Brochu E, Rioux I, LeBlanc M, Daley M, Morin C (2003) Cognitive performance and sleep quality in the elderly suffering from chronic insomnia. Relationship between objective and subjective measures. J Psychosom Res 54:39-49.

Brower KJ (2001) Alcohol's effects on sleep in alcoholics. Alcohol Res Health 25:110-125.

Brower KJ (2003) Insomnia, alcoholism and relapse. Sleep Med Rev 7:523-539.

Brower KJ, Aldrich M, Robinson EAR, Zucker RA, Greden JF (2001) Insomnia, self-medication, and relapse to alcoholism. Am J Psychiatry 158:399-404.

Brower KJ, Aldrich MS, Hall JM (1998) Polysomnographic and subjective sleep predictors of alcoholic relapse. Alcohol Clin Exp Res 22:1864-1871.

Buysse DJ, Reynolds CF 3rd, Monk TH, Berman SR, Kupfer DJ (1989) The Pittsburgh sleep quality index: a new instrument for psychiatric practice and research. Psychiatry Res 28:193-213.

Caetano R, Clark CL, Greenfield TK (1998) Prevalence, trends, and incidence of alcohol withdrawal symptoms: analysis of general population and clinical samples. Alcohol Health Res World 22:73-79.

Carskadon M, Dement W, Mitler M, Guilleminault C, Zarcone V, Spiegal R (1976) Self reports versus sleep laboratory findings in 122 drugfree subjects with complaints of chronic insomnia. Am J Psychiatry 12:1382-1388

Clark C, Gillin J, Golshan S, Demodena A, Smith T, Danowski S, Irwin MMS (1998) Increased REM sleep density at admission predicts relapse by three months in primary alcoholics with a lifetime diagnosis of secondary depression. Biol Psychiatry 43:601-607.

Cohen J (1988) Statistical Power Analysis for the Behavioral Sciences. Erlbaum, Hillsdale, NJ.

Cohn T, Foster J, Peters T (2003) Sequential studies of sleep disturbance and quality of life in abstaining alcoholics. Addict Biol 8:455-462.

Currie S, Malhotra S, Clark S (2004) Agreement among subjective, objective, and collateral measures of insomnia in postwithdrawal recovering alcoholics. Behav Sleep Med 2:148-161.
Dorsey C, Bootzin R (1997) Subjective and psychophysiologic insomnia: an examination of sleep tendency and personality. Biol Psychiatry 41:209-216.

Douglass AB, Bornstein R, Nino-Murcia G, Keenan S, Miles L, Zarcone V Jr., Guilleminault C, Dement WC (1994) The sleep disorders quesetionnaire I: creation and multivariate structure of SDQ. Sleep 17:160-167.

Drummond S, Gillin J, Smith T, DeModena A (1998) The sleep of abstinent pure primary alcoholic patients: natural course and relationship to relapse. Alcohol Clin Exp Res 22:1796-1802.

Edinger JD, Fins AI (1995) The distribution and clinical significance of sleep time misperceptions among insomniacs. Sleep 18:232-239.

Edinger J, Fins A, Glenn M, Sullivan R, Bastian L, Marsh G, Dailey D, Hope T, Young M, Shaw E, Vasilas D (2000) Insomnia and the eye of the beholder: are there clinical markers of objective sleep disturbances among adults with and without insomnia complaints? J Consult Clin Psychol 68:586-593.

Foster J, Marshall E, Peters T (1998) Predictors of relapse to heavy drinking in alcohol dependent subjects following alcohol detoxification: the role of quality of life measures, ethnicity, social class, cigarette and drug use. Addict Biol 3:333-343.

Foster J, Marshall E, Peters T (2000) Application of a quality of life measure, the life situation survey (LSS), to alcohol dependent subjects in relapse and remission. Alcohol Clin Exp Res 24:1687-1692.

Foster J, Peters T (1999) Impaired sleep in alcohol misusers and dependent alcoholics and the impact upon outcome. Alcohol Clin Exp Res 23:1044-1051.

Frankel B, Coursey R, Buchbinder R, Snyder F (1976) Recorded and reported sleep in chronic primary insomnia. Arch Gen Psychiatry 33:615-623.

Freemon F (1982) The effect of chronically administered delta-9-tetrahydrocannabinol upon the polygraphically monitored sleep of normal volunteers. Drug Alcohol Depend 10:345-353.

Gann H, Feige B, Hohagen F, van Calker D, Geiss D, Dieter R (2001) Sleep and the cholinergic rapid eye movement sleep induction test in patients with primary alcohol dependence. Biol Psychiatry 50:383-390.

Gann H, van Calker D, Feige B, Cloot O, Bruck R, Berger M, Riemann D (2004) Polysomnographic comparison between patients with primary alcohol dependency during subacute withdrawal and patients with a major depression. Eur Arch Psychiatry Clin Neurosci 254:263271.

Gillin J, Smith T, Irwin M, Butters N, Demodena A, Schuckit M (1994) Increased pressure for rapid eye movement sleep at time of hospital admission predicts relapse in nondepressed patients with primary alcoholism at 3 month follow up. Arch Gen Psychiatry 51:189-197.

Guy W (1976) Clinical Global Impressions. National Institute of Mental Health, Rockville, MD.

Hoffmann R, Emslie G, Thompson J, Rintelmann J, Moore J, Armitage $\mathrm{R}$ (2004) The relationship between actigraphy and polysomnography in healthy children and adolescents. Sleep 27:A816.

Jenkins C, Stanton B, Niemcryk S, Rose R (1988) A scale for the estimation of sleep problems in clinical research. J Clin Epidemiol 41: 313-321.

Kales A, Kales J (1984) Evaluation and Treatment of Insomnia. Oxford, New York.

Means M, Edinger J, Glenn M, Fins A (2003) Accuracy of sleep perceptions among insomnia sufferers and normal sleepers. Sleep Med 4: 285-296.

Mello NK, Mendelson JH (1970) Behavioral studies of sleep patterns in alcoholics during intoxication and withdrawal. J Pharmacol Exp Ther 175:94-112.

Monroe L (1967) Psychological and physiological differences between good and poor sleepers. J Abnorm Psychol 72:255-264.

Morin CM (1993) Insomnia Psychological Assessment and Management. The Guilford Press, New York.

NIAAA (2005) A Pocket Guide For Alcohol Screening and Brief Intervention, in Helping Patients Who Drink Too Much: A Clinician's 
Guide, 2005 ed. NIAAA Publications Distribution Center, Rockville, MD.

Rechtschaffen A, Kales AA (1968) A Manual of Standardized Terminology, Techniques and Scoring System for Sleep Stages of Human Subjects. Government Printing Office, Washington, DC.

Rosa R, Bonnet M (2000) Reported chronic insomnia is independent of poor sleep as measured by electroencephalography. Psychosom Med 62:474-482.

Rundell OH, Williams HL, Lester BK (1977) Sleep in alcoholic patients: longitudisnal findings. Adv Exp Med Biol 85B:389-402.
Skoloda T, Alterman A, Gottheil E (1979) Sleep Quality Reported by Drinking and Non-Drinking Alcoholics. Pergamon Press, Elmsford, NY.

Sobell L, Sobell M, Leo G, Cancilla A (1988) Assessing normal drinkers' reports of recent drinking and a comparative evaluation across several populations. Br J Addict 83:393-402.

Sullivan JT, Sykora K, Schneiderman J, Naranjo CA, Sellers EM (1989) Assessment of alcohol withdrawal: the revised clinical institute withdrawal assessment for alcohol scale (CIWA-Ar). Br J Addict 84: $1353-1357$. 